Las formas de la vitalidad en la recepción de una obra teatral.

Alicia Clara Nudler, María de la Paz Jacquier, Silvia Español

Epistemus - Revista de estudios en Música, Cognición y Cultura, 8(1), 1-24, e013, 2020

ISSN 1853-0494 | https://doi.org/10.24215/18530494e013

https://revistas.unlp.edu.ar/Epistemus

Sociedad Argentina para las Ciencias Cognitivas de la Música (SACCoM)

Universidad Nacional de La Plata

La Plata | Buenos Aires | Argentina

\title{
Las formas de la vitalidad en la recepción de una obra teatral
}

\author{
Alicia Clara Nudler', María de la Paz Jacquier ${ }^{2}$ y Silvia Español ${ }^{3}$ \\ anudler@gmail.com \\ 1,2Universidad Nacional de Río Negro. \\ ${ }^{3}$ Facultad Latino Americana de Ciencia Sociales (FLACSO). Consejo Nacional de Investigaciones \\ Científicas y Técnicas (CONICET).
}

\section{Resumen}

En el artículo se describe un estudio empírico realizado con espectadores de una obra de teatro-danza, cuyo objetivo fue explorar la captación de formas de la vitalidad (Stern, 1985 y 2010). El estudio se caracteriza por un diseño complejo que implicó (a) la puesta en escena de una obra teatral, (b) la conformación de un estímulo novedoso a partir de la obra, y (c) la creación de tareas a ser realizadas por los espectadores al finalizar la función. La obra a la que asistieron los espectadores/participantes fue Dibaxu del director Hugo Aristimuño. El estímulo creado fue la proyección de dos fragmentos de la obra filmados en vivo en una de las funciones. Las tareas fueron cuatro: dos tareas no-verbales, originales y novedosas, y dos verbales. Se describen los rasgos particulares de la obra, el proceso de creación del estímulo y las tareas creadas. Se presentan los resultados de la tarea verbal que consistió en la selección de palabras descriptoras de formas de la vitalidad para caracterizar los fragmentos seleccionados de la obra presenciada. Se comentan luego los desafíos metodológicos del estudio de la recepción de espectáculos en vivo, se realizan comparaciones con otros trabajos del campo, y se resalta la virtud del diseño que permitió crear un estímulo breve que conserva, sin embargo, las cualidades de la obra completa. Finalmente, se discute la contribución de la investigación realizada tanto al estudio de las formas de la vitalidad como a los estudios de públicos de teatro y danza de orientación cognitiva.

\section{Palabras Clave}

Formas de la vitalidad, teatro-danza, recepción, cognición corporizada. 


\title{
Forms of vitality in the reception of a theater work
}

\begin{abstract}
This article describes an empirical study conducted with spectators of a theater-dance piece with the goal of exploring the reception of forms of vitality (Stern, 1985 and 2010). The study entails a complex design including a) the production of a live performance; b) the creation of an original stimulus based on the play; c) the design of tasks to be carried by spectators after the show. The performance attended by the spectators/participants was Dibaxu by theater director Hugo Aristimuño. The stimulus was the projection of two excerpts of the play recorded during one of the live performances. The tasks were four: two non-verbal tasks, original and innovative, and two verbal ones. The specific traits of the performance, the process of creation of the stimulus and the tasks carried by participants are described. The results are presented of the verbal task consisting in the selection by participants of forms of vitality words to describe the excerpts. The article also discusses the challenges of studying the reception of live performances, establishes comparisons with other studies in the field, and points to the advantage of designing a short stimulus which nevertheless retains some of the qualities of the whole performance. Finally, it discusses the contribution of the study to the knowledge of forms of vitality and to cognitively oriented research on dance and theater audiences.
\end{abstract}

\section{Key Words}

Forms of vitality, theater-dance, reception, embodied cognition.

\section{Introducción4}

En este trabajo se relata una exploración empírica de la captación de las formas de la vitalidad por parte de espectadores de una obra de teatro-danza. Se desarrolló un diseño específico para indagar a través de herramientas lingüísticas y no lingüísticas la experiencia de la vitalidad de los espectadores de una obra que despliega movimientos coreográficos en un marco narrativo. El trabajo pretende contribuir al creciente conocimiento empírico de las formas de la vitalidad generado a partir de que Daniel Stern $(1985,2010)$ acuñara el término para describir una cualidad específica de la experiencia intersubjetiva y con las artes temporales. La investigación es además un aporte a los estudios de orientación cognitiva con espectadores de teatro y danza.

Stern (1985) propuso el concepto de afectos de la vitalidad en el marco de sus 
estudios de desarrollo temprano para describir una cualidad de la experiencia que "puede surgir directamente del encuentro con la gente, una cualidad que envuelve afectos energéticos" (p. 75). Consideró necesario acuñar esa nueva expresión ya que muchas cualidades emocionales no se ven reflejadas en otras taxonomías de afectos. Los afectos de la vitalidad se ven suscitados en el infante por actos parentales generalmente no considerados como actos afectivos regulares: el modo en que la madre alza al bebé, pliega los pañales, etc. Los afectos de la vitalidad involucran el cómo se realizan las acciones, no las acciones mismas. Stern diferenció además esta experiencia de los afectos darwinianos (alegría, tristeza, etc.), señalando que esas cualidades elusivas de la experiencia son aprehendidas por términos dinámicos, cinéticos, tales como agitación, desvanecimiento progresivo, fugaz, etc.

El autor consideró la percepción de estos afectos en los dos primeros meses de vida -la etapa del sí mismo emergente- como uno de los recursos tempranos que permiten la emergencia de la primera organización psíquica. Destacó también su importancia en el período del sí mismo subjetivo, entre los nueve y los quince meses, donde los afectos de la vitalidad juegan un rol clave en el entonamiento afectivo. El entonamiento afectivo es un concepto también acuñado por Stern para designar un tipo especial de conducta materna que el autor identificó en sus numerosas observaciones de interacciones madre-bebé: son conductas de imitación de los afectos de la vitalidad o rasgos temporales de la conducta del bebé -el ritmo, por ejemplo- que la madre despliega con el solo fin de comunicar sintonía con el bebé (Stern, 1985). Esas conductas maternas -el entonamiento afectivo- tienen un rol crucial en el establecimiento de las experiencias de intersubjetividad.

Si bien Stern propuso dicho concepto para referirse específicamente a las conductas de imitación de los afectos de la vitalidad de los bebés por parte de los adultos, en obras posteriores tales como The Present Moment in Psychotherapy and Everyday Life (2004) y en su último libro Forms of Vitality (2010) parece dejar abierta la posibilidad de que estos fenómenos sucedan también entre adultos. Otros autores también así lo consideran (por ejemplo, Shifres, Pereira Ghiena, Herrera y Bordoni, 2012). El entonamiento afectivo es tomado como concepto por fuera del contexto adulto-bebé en el campo de la musicoterapia; por ejemplo Trondalen y Skårderud (2007) señalan que el entonamiento afectivo reviste una importancia crucial tanto en la interacción madre-infante como en la relación terapeutapaciente, y ofrecen una viñeta clínica del tratamiento con un joven con anorexia donde describen entonamientos mutuos entre terapeuta y paciente en cada uno de los aspectos señalados por Stern (intensidad absoluta, perfil de la intensidad, pulsación, ritmo, duración, y pauta espacial).

Los afectos de la vitalidad, entonces, son una experiencia subjetiva que puede ser expresada y compartida con los demás, y por eso tienen un papel destacado 
en los fenómenos de entonamiento que, parafraseando a Shifres, Pereira Ghiena, Herrera y Bordoni (2012) suceden

"cuando en vez de imitar -es decir, emparejar la totalidad de la conducta- respondemos emparejando ciertos aspectos amodales o supramodales de la misma, generalmente transportándolos a otra modalidad sensorial (por ejemplo, transponer el ritmo de una vocalización al ritmo de un movimiento de brazos)" (p. 91).

En 2010, Stern renombró los afectos de la vitalidad como "formas dinámicas de la vitalidad" (p. 3) y las definió con mayor precisión como "fenómenos subjetivos, psicológicos, que emergen del encuentro con los eventos dinámicos" (2010, p. 7, trad. de los autores), Gestalts que surgen de las experiencias de movimiento, fuerza, tiempo, espacio e intención. Las formas dinámicas de la vitalidad informan sobre el estado subjetivo de las personas.

Varios trabajos han indagado empíricamente diversos aspectos de las formas de la vitalidad, y a continuación citamos algunos de ellos, a modo de ofrecer un panorama del campo en la actualidad, que no pretende ser exhaustivo. Rochat, Veroni, Bruschweiler-Stern, Pieraccini, Bonnet-Brilhaultet al, (2013), por ejemplo, diferenciaron el qué y el cómo de ciertas acciones solicitando a una serie de personas de desarrollo típico y otras con síndrome autista que distingan entre acciones simples efectuadas de dos modos distintos -fuerte o suave- en distintas combinaciones. Uno de los resultados más llamativos que obtuvieron fue que las personas autistas cometieron una cantidad significativamente mayor de errores que las de desarrollo típico en la tarea de reconocer la misma forma de la vitalidad en acciones diferentes, o distintas formas de la vitalidad en la misma acción (este mismo error no se repitió en la tarea de distinguir acciones diferentes). Los investigadores arribaron a la conclusión de que la captación del cómo se realizan las acciones, o la captación de la forma de la vitalidad que conllevan, es una habilidad específica que se encuentra alterada en algunas patologías. Este estudio tiene en común con el nuestro el centrarse en la captación de las formas de la vitalidad; sin embargo, en él los estímulos despliegan formas opuestas entre sí (fuerte-suave) en un diseño simple, en función del objetivo de estudiar las diferencias entre sujetos de condición típica y sujetos del espectro autista. Otra diferencia importante es que en el estudio de Rochat y otros no interviene el rótulo lingüístico de las formas de la vitalidad, los sujetos sólo tenían que reconocer diferencias y semejanzas seleccionando el símbolo igual o el símbolo diferente. Citamos este trabajo solamente a modo de ejemplo de trabajos empíricos que han indagado la captación de formas de la vitalidad, que muestran que el modo en que se realizan las acciones es un elemento importante y distinguible en su percepción.

Shifres, Pereira Ghiena, Herrera y Bordoni (2012), en un trabajo sobre estilos 
en el tango, mostraron que las formas de la vitalidad, al ser de naturaleza amodal, permiten una continuidad estilística a través de diversas modalidades perceptuales por las que el contenido dinámico puede atravesar. Los autores exploraron empíricamente dicha continuidad, tomando como motivo de estudio el tango en su aspecto musical y de danza. A través de un complejo diseño experimental, mostraron que el estilo de la orquesta al interpretar un tango se transponía al baile de una pareja de tango, de modo que los estilos terminaban siendo diferenciables entre sí por sujetos que sólo eran expuestos a la condición visual de una filmación del baile sin escuchar la música. El estudio exploró específicamente la transmodalidad de las formas de la vitalidad, i.e. cómo se traslada determinada cualidad de lo auditivo a lo visual. En el estudio que aquí presentamos, la transmodalidad de las formas de la vitalidad no fue objeto de estudio, sino que se la tomó como un supuesto.

Shifres (2008) presenta un trabajo basado en el análisis de un fragmento musical ejecutado en la película El Pianista de Roman Polansky. La similitud de este trabajo con el nuestro es que se tomó un fragmento de una pieza dramática, es decir, un estímulo audiovisual en el marco de una narración. Aunque los sujetos del estudio de Shifres no necesariamente habían visto la película, se les relataba la historia. De este modo se discuten las formas de la vitalidad como cualidad sentida e intermodal de la experiencia en un marco narrativo. Este trabajo arroja un resultado sumamente interesante: la narrativa que envuelve la percepción de formas de la vitalidad puede influir la captación de esas formas.

De todos los estudios empíricos sobre formas de la vitalidad relevados (entre otros, además de los ya citados, Di Cesare, Di Dio, Rochat, Sinigaglia, Bruschweiler-Stern, et al., 2013; Español y Bordoni, 2018; Español, Bordoni, Martínez y Carretero, 2015) el que guarda más similitudes con el nuestro es el de Martínez y Pereira Ghiena (2011), ya que involucró categorías lingüísticas así como movimiento de los propios sujetos. Los investigadores partieron del supuesto de que las formas de vitalidad emergentes de la música y el movimiento pueden "(i) ser interpretadas como descripciones no lingüísticas mediante el movimiento corporal y (ii) asociarse a las descripciones lingüísticas que dan cuenta de esa calidad vital" (p. 3). En nuestro trabajo también suponemos un correlato entre la cualidad sensorio-motriz de la experiencia y un descriptor lingüístico. Los autores, apoyados en la teoría de Leman (2008), plantean que cuando los sujetos se mueven durante la recepción musical, como ocurre en una de las condiciones experimentales, están realizando una descripción musical no lingüística. Similarmente al trabajo de Martínez y Pereira Ghiena, el movimiento podría entenderse como una descripción no lingüística de la percepción de la escena teatral. Una diferencia entre ambos trabajos reside en la constitución de las condiciones experimentales: mientras que Martínez y Pereira Ghiena establecen 5 condiciones diferenciadas principalmente por la modalidad perceptual (auditiva, audiovisual, kinético-auditiva y visual), las 
condiciones en nuestro trabajo están dadas por dos estímulos contrastantes en cuanto a sus formas dinámicas de la vitalidad preponderantes (dos fragmentos de una misma obra de danza-teatro). Y otra diferencia importante es que el diseño experimental sobre la obra teatral plantea una tarea de movimiento explícito, y los movimientos de los sujetos constituyen parte de los resultados, mientras que el trabajo citado utiliza el movimiento de los sujetos como parte de una de las condiciones en las que se realiza la prueba, pero no analiza el tipo de movimientos producidos. Se retoma la referencia a este trabajo en el apartado Discusión.

Como señalan Nudler y Jacquier (2018), las formas dinámicas de la vitalidad, este aspecto de la experiencia y la conducta humanas clave para la comprensión de la intersubjetividad, tienen un papel destacado en la comunicación que se produce en el teatro, en tanto arte temporal que transmite sus sentidos profundos a través del cuerpo y la voz de los actores, y del juego en el tiempo de sus elementos kinéticos, sonoros y visuales.

En cuanto a estudios de formas de la vitalidad específicamente en el teatro, son pocos los trabajos con los que se cuenta. Stern trabajó junto al director Robert Wilson, arribando a la obra Bob's breakfast: From the mind to the stage (citada en Stern, 2010), obra aún inédita que mayormente describe tipos de movimientos y desplazamientos con sus correspondientes cualidades. Si bien ésta es una sugerente primera exploración de las formas de la vitalidad en el teatro, aparece inconclusa e invita a la realización de estudios más exhaustivos.

Nudler, Español, Jacquier y Porcel de Peralta (2018), por su parte, realizaron un análisis de formas dinámicas de la vitalidad en un fragmento escogido de tres versiones distintas de la obra La última cinta de Krapp del dramaturgo Samuel Beckett (1986). En este análisis, utilizando un programa para análisis de video, compararon formas de la vitalidad en cuatro dimensiones: movimientos del cuerpo del actor, voz, otros sonidos de la escena e iluminación; crearon subcategorías basadas en atributos descriptos por Stern -por ejemplo repentino, suave, esforzado- y las asignaron a cada uno de los fragmentos y versiones a lo largo de la línea temporal, logrando así dar cuenta de cómo las diferentes formas de la vitalidad y sus diversas combinaciones generaban distintas características psicológicas del personaje y distintas experiencias en el espectador, aun cuando el parlamento del personaje fuera el mismo.

Bussières (2018), una iluminadora teatral, considera que la concepción de vitalidad de Stern es fundamental para comprender el posible dinamismo de la luz. En su novedoso trabajo sobre el uso de iluminación teatral, analiza la luz como fenómeno temporal y dinámico en diferentes períodos y poéticas teatrales y sus efectos en los espectadores.

Nuestro trabajo guarda por otro lado similitudes con estudios realizados en 
el marco del proyecto The Watching Dance Project (2008-2011) desarrollado entre varias universidades del Reino Unido. Este proyecto llevó a cabo estudios cualitativos con pequeños números de individuos, con diversas técnicas lingüísticas y no lingüísticas, buscando obtener una comprensión compleja y detallada de sus experiencias y respuestas al presenciar una performance. Trabajaron en torno al concepto de empatía kinestésica: lo que sucede cuando los espectadores de danza, aún sentados en sus butacas, sienten que están participando en los movimientos que observan, y experimentan sensaciones e ideas relacionadas. El estudio que aquí se presenta guarda relación con estos trabajos porque supone también una resonancia corporal en los espectadores como producto del movimiento de los actores en escena. En la introducción al libro que resume varios de los resultados de ese proyecto, Jones (2012) se pregunta: “¿Cómo llegan los cuerpos, especialmente aquellos creativamente motivados para moverse con el fin de comunicar sentido artístico, a significar para los demás?” (p. 14, traducción de los autores). Esta es en última instancia la pregunta que se encuentra también en el horizonte de nuestro trabajo.

Más allá de esta serie de trabajos que citamos, todos dentro de la temática, hasta donde sabemos no existen estudios empíricos de captación de formas de la vitalidad por parte de espectadores de teatro. El estudio que referimos aquí es por lo tanto el primero de este tipo.

\section{Método}

\section{Diseño}

Se realizó este estudio con la intención de indagar la experiencia de la recepción de las formas de la vitalidad de los espectadores de una obra de danza-teatro. La obra seleccionada fue Dibaxu del director teatral Hugo Aristimuño, basada en poemas de Juan Gelman. La obra relata momentos de la vida del poeta durante la dictadura en Argentina (1976-1983), especialmente la historia de sus hijos desaparecidos, y su propio exilio posterior. Antes de que comience el espectáculo propiamente dicho, se escucha un texto del Libro de los Abrazos de Eduardo Galeano (2013) donde se hace referencia a esos aspectos de la vida de Gelman, lo cual enmarca sentidos de la obra desde el inicio. La obra contiene texto escrito por el director Aristimuño a partir de poemas de Gelman y de entrevistas que aparecieron en los medios de comunicación. Asimismo, la obra tiene gran despliegue de movimiento coreografiado. Hay una importante presencia del componente sonoro; la utilización de ciertos objetos y el vestuario son especialmente cuidados.

La puesta para este estudio, así como las anteriores, estuvo a cargo del grupo 
que dirige Aristimuño, Teatro del Viento, de la ciudad de Viedma, siendo los actores-bailarines Débora Sansó, Carlos Irazusta y Guillermo Riegelhaupt.

Se eligió esta obra para la realización de este estudio por criterios estéticos e ideológicos, y porque es una obra con importante despliegue de movimiento estético expresivo donde además hay un relato que enmarca y da sentido a esos momentos coreográficos. Por otro lado, esta obra había sido mostrada a público en varias ciudades del país y del exterior en incontables oportunidades con anterioridad, siempre con muy buena recepción y críticas favorables en los medios. Consideramos esto un factor importante, por un lado porque al estar la producción a cargo del equipo de investigación debíamos asegurarnos la afluencia de público, y por el otro porque ofrecía cierta garantía de que los espectadores se mantendrían interesados por la performance a lo largo de toda su duración, cosa que efectivamente sucedió. Por último la obra se eligió también por razones de factibilidad tanto técnica como de posibilidades de establecer acuerdos con el elenco, necesarios para poder realizar el estudio.

Los participantes del estudio presenciaron la obra durante la función e inmediatamente después observaron un fragmento breve de la obra, al que le sucedieron diversas tareas: a. Estación de movimiento; b. Estación de dibujos; c. Estación de palabras; d. Estación de círculo verbal.

El estudio supuso dos condiciones. En cada una de ellas el fragmento principal de la obra elegido para la realización de las tareas de los participantes fue diferente. (Ver la descripción de los fragmentos en el apartado Estímulos).

De los 24 participantes, 12 estuvieron expuestos a la Condición A y 12 a la Condición B.
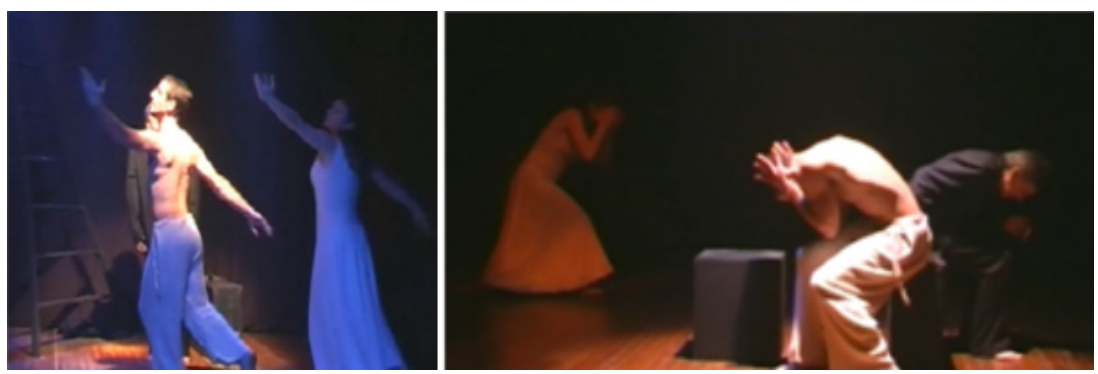

Figura 1. Instantáneas del Fragmento 1 (izquierda) y del Fragmento 2 (derecha) respectivamente. 


\section{Participantes}

Participaron de este estudio 24 personas adultas, que no habían visto la obra Dibaxu anteriormente. El rango etario osciló entre los 18 y los 71 años. Doce participantes fueron estudiantes del Profesorado de Teatro o de la Licenciatura en Arte Dramático de la Universidad Nacional de Río Negro, y 12 fueron profesionales, empleados y otros estudiantes, no vinculados particularmente al teatro. Los participantes estudiantes y no estudiantes se repartieron de forma igual entre ambas condiciones para evitar un sesgo en la resolución de la tarea 2 (movimiento) considerando que era probable que los estudiantes de teatro tuvieran más facilidad para el movimiento. Sin embargo, no se implementó un diseño comparativo de estudiantes y no estudiantes.

\section{Aparatos y materiales}

En la Estación a se utilizó un equipo de audio con parlantes externos para reproducir las piezas musicales. Las instancias de movimiento fueron filmadas con dos cámaras: una para la captura del grupo en su totalidad, y otra para la captura de los participantes individualmente. Para la Estación b se utilizaron diversos materiales de librería: papel afiche y papel fotográfico de 60 x $90 \mathrm{~cm}$, crayones, fibras, fibrones, acuarelas, lápices, biromes de diversos colores. Para la Estación c se utilizaron 9 netbooks, con sus respectivos auriculares, que contaban con programas para la reproducción de los fragmentos escénicos. Allí, los datos fueron registrados por los participantes en planillas impresas.

\section{Estímulos}

Los estímulos principales fueron la obra propiamente dicha, representada en el teatro para un público general, y los dos fragmentos de la misma obra que se filmaron en vivo el día sábado, luego se recortaron y se proyectaron de manera inmediatamente posterior a la función de cada día (el Fragmento 1 se proyectó el día sábado, el Fragmento 2 el día domingo). Para este estudio, el fragmento recortado sólo cobra sentido como estímulo en la medida en que es presenciado en el marco de la obra total. De este modo, el estímulo construido logra una suerte de compromiso entre la indagación de la recepción de un fragmento breve (lo que permite repetidas reproducciones y análisis minucioso) y la experiencia global y sumamente compleja que implica presenciar una obra teatral. Confeccionado de este modo, el estímulo logra condensar, expresar, y repetir momentos significativos de la recepción de la obra.

La selección de los fragmentos que se utilizarían la realizó el equipo de investigación con anterioridad al estudio en base a filmaciones de puestas anteriores de la obra. Para ello los siete integrantes del equipo de investigación observaron 
iterativamente la filmación de toda la obra. Se hizo una primera selección de seis fragmentos y de ellos se seleccionaron dos fragmentos en los que hubo acuerdo entre todos respecto de que eran claramente contrastantes, a la vez que expresaban formas de la vitalidad preponderantes a lo largo de toda la obra (ver la caracterización más abajo). En los dos fragmentos utilizados hay movimiento de los actores-bailarines, hay música, y no hay texto hablado. Ambos condensan algo del dramatismo del relato que se cuenta, como así también proponen una lectura rica en signos, y clara desde el punto de vista narrativo.

El Fragmento 1 comienza a partir del minuto 4 aproximadamente de transcurrida la obra y tiene una duración de 1 minuto. Una luz frontal ilumina la escena que comienza en silencio. Los tres personajes inician un relato con una respiración coordinada, luego irrumpe la música. Con movimientos convergentes con la música señalan hacia el frente de la escena y comienzan una actividad más dinámica, realizando numerosos giros y movimientos más bien ligados. Dos de ellos se ubican en un plano narrativo abierto y frontal como avistando un futuro; el tercer personaje, detrás, funciona como generador de eso que viven los personajes de adelante. Al final del fragmento el tercer actor produce un corte abrupto como despertándolos de un sueño. Así, el baile culmina con un salto y caída también abruptos.

La música que sonoriza esta escena forma parte del Concierto para Dos Cellos en Sol menor, RV 531 de Antonio Vivaldi, interpretado por Bobby McFerrin (voz), Veronika Wilhelm (cello), y la Gewandhausorchester de Leipzig. La música también parece girar, si seguimos la idea de una composición contrapuntística que va entrelazando las ideas musicales, como direccionándose siempre hacia adelante, con fuerza y en un tempo moderado a rápido. La versión de Bobby McFerrin, aunque fiel a la original, agrega extrañamiento e interés a la pieza musical.

El Fragmento 2 comienza a los 5 minutos y 30 segundos de iniciada la obra, y tiene una duración de 1 minuto y 30 segundos. Una luz cenital ilumina a los dos actores que se encuentran de frente al público y a una actriz de espalda desplazada a un lado de la escena. Luego de un instante de mínimos movimientos continuos coordinados y en cámara lenta, al igual que en el Fragmento 1 una profunda respiración marca el inicio del relato de la escena con movimientos convergentes con la música. A través de expresiones enfáticas y sorpresivas cada actor cuenta algo que trasunta aspectos intensamente dramáticos. En este fragmento la respiración agrega sonoramente un carácter trágico a la escena. El Fragmento 2 es mucho más lento, contenido y tenso que el Fragmento 1, con algunos movimientos cortados y rápidos.

El Fragmento 2 está sonorizado por parte de la canción Boga, interpretada por Amiina, que es el tema 12 del álbum Kurr (2007). Aquí la música es lenta, con 
melodías superpuestas pero simples. Predominan los sonidos largos, una tímbrica metálica, las armonías en modo menor y los clusters, subrayando la tensión de la escena.

En la Estación a (movimiento) se utilizaron las piezas completas que sonorizan cada uno de los fragmentos en la obra.

\section{Procedimiento}

Al contactar a potenciales participantes en el estudio, se les informó brevemente de qué se trataba la investigación, y se les anticipó que, si aceptaban participar, deberían permanecer en la sala para realizar una serie de actividades una vez finalizado el espectáculo.

Ambos días de las funciones, previamente a iniciar el espectáculo, los participantes completaron una planilla con datos personales y firmaron el consentimiento informado antes de ingresar a la sala. A continuación presenciaron la función desde sus butacas reservadas para ellos en las filas primera y segunda de la sala. Al concluir la obra, se les pidió a los participantes que permanecieran en la sala, e inmediatamente después se proyectó el Fragmento 1 en la Condición A (día sábado), y el Fragmento 2 en la Condición B (día domingo). Luego de esta proyección, se los invitó a realizar las tareas de las distintas Estaciones, a saber:

a. Estación de movimiento. Las personas pasaban al escenario, delimitado por el telón del proscenio y un telón intermedio, lo que generaba un espacio circunscripto no demasiado pequeño (aproximadamente de 7 x 3 metros). En el escenario sonaba de manera continua la música que correspondía al fragmento que acababan de ver en la obra teatral. El coordinador de esta estación, uno de los investigadores, invitaba a estas 12 personas a moverse y desplazarse libremente por el espacio, y les sugería, en un tono suave de voz, mantener el estado que esta obra y/o el fragmento recién presenciado les hubieran generado. Al cabo de algunos minutos los invitaba a que, en la medida que cada uno lo deseara, pasaran de manera individual al espacio contiguo (un espacio más pequeño, delimitado por el telón intermedio y la pared del fondo, suavemente iluminado y donde también se escuchaba el sonido de la música); les pedía que allí, a partir del sedimento que la obra había dejado en su propia experiencia, cada uno imprimiera un gesto o un movimiento durante un tiempo aproximado de 40 segundos (estos movimientos fueron filmados por una cámara fija que estaba oculta (aunque ellos sabían de antemano que se los estaba filmando, la cámara se ocultó para evitar un posible efecto de inhibición). Las personas al salir del cuarto contiguo permanecían con el resto del grupo en el espacio más amplio, contribuyendo a mantener el particular estado generado. Una vez que los 12 participantes habían pasado al espacio individual a imprimir su movimiento, se los invitaba a pasar a la próxima estación. 
b. Estación de dibujos. La consigna aquí fue que dejaran una huella en hojas de papel, es decir, que con los materiales disponibles plasmaran algunas de sus sensaciones al percibir el fragmento de la obra. Podían elegir utilizar las hojas de papel de modo vertical (colocadas en pizarrones) o de modo horizontal, en el suelo o en mesas. Se les pidió que identificaran sus producciones con un nombre. A medida que concluían, pasaban a la próxima estación.

c. Estación de palabras. Cada participante contaba con una netbook y auriculares. Se les pidió que vieran nuevamente el fragmento que se les había proyectado en pantalla gigante ni bien finalizada la función (Fragmento 1 para la Condición A, Fragmento 2 para la Condición B), ahora en sus netbooks individuales. Luego, en una planilla que contenía un listado de setenta y cinco palabras descriptoras de formas de la vitalidad, tenían que marcar todas aquellas palabras que para ellos describieran dicho fragmento. También podían agregar palabras que no estuvieran en la lista. Podían reproducir el fragmento cuantas veces quisieran para completar la planilla. Al finalizar, entregaban la planilla con las palabras elegidas para el Fragmento 1, y se les entregaba la planilla 2, que contenía las mismas setenta y cinco palabras, que ahora tenían que aplicar al otro fragmento (Fragmento 2 para la Condición A, Fragmento 1 para la Condición B). Es decir que en cada condición los sujetos correlacionaron palabras con ambos fragmentos, pero primero realizaron la tarea con el fragmento que habían visto en pantalla gigante y cuya música sonó durante la Estación a (movimiento), y luego con el otro fragmento.

El corpus de setenta y cinco palabras fue creado por el equipo de investigación a partir de tres fuentes: a) el listado de palabras brindadas por Stern (2010) compuesto por treinta y tres palabras, mayormente adverbios y gerundios, al final del cual se lee "y muchas más" (p. 7). De este listado se seleccionaron y tradujeron palabras de modo de que resultaran relativamente comunes, a la vez conservando el sentido del original en inglés; b) otras palabras citadas en la literatura sobre formas de la vitalidad; c) otras listas de adjetivos y gerundios tomadas de clases de contact-improvisación de las que el equipo seleccionó aquellos que mejor describían contornos dinámicos. El corpus se armó de este modo al no contarse en la actualidad con un repositorio consensuado de palabras descriptoras de formas de la vitalidad. Existe una dificultad en seleccionar palabras descriptoras de formas de la vitalidad, ya que estos contornos vitales son captados generalmente de modo implícito, no verbal, y son además potencialmente infinitos. Se seleccionaron muchas palabras para poder reflejar la sutileza de las diferencias, aunque esto devino un problema, que se hizo evidente al momento de analizar los resultados, y que retomamos en la discusión.

Al cabo de las tres primeras estaciones (movimiento, dibujos, palabras) se les solicitó completar una encuesta con impresiones acerca de la obra. 
d. Estación en círculo. Finalmente, mientras se les ofreció un refrigerio, se los invitó a compartir sus impresiones en un intercambio grupal en círculo coordinado por uno de los miembros del equipo, donde también participó el resto del equipo.

Las Estaciones a, b y d son propuestas originales ideadas por los investigadores para estudiar la captación de formas de la vitalidad en la experiencia de presenciar teatro. La Estación c consiste en una técnica de recolección de datos frecuentemente implementada en estudios psicológicos y que ha sido utilizada también para indagar la captación de las formas de la vitalidad, por ejemplo en el ya citado Martínez y Pereira Ghiena (2011).

El procedimiento descrito fue realizado de igual modo el día sábado (Condición A) y el día domingo (Condición B). La diferencia entre ambos consistió en que el sábado se proyectó en pantalla gigante el Fragmento 1, y la música que sonó en la Estación a (movimiento) fue la pieza de Vivaldi interpretada por Bobby McFerrin, es decir, la correspondiente a dicho fragmento, y por lo tanto la Estación a (movimiento) y la Estación b (dibujo) estuvieron más impregnadas de las formas de la vitalidad de ese fragmento. Mientras que el día domingo se proyectó el Fragmento 2, y la música fue Boga de Amiina, es decir la correspondiente al Fragmento 2. Para la Estación c (palabras), los del día sábado (Condición A) primero trabajaron con el Fragmento 1 y luego con el 2, mientras que los del día domingo (Condición B) lo hicieron en el orden inverso.

\section{Resultados}

De los datos obtenidos en las cuatro estaciones (movimiento, dibujos, palabras y círculo verbal), en este artículo se presentan y discuten únicamente los resultados encontrados en la Estación c (palabras), en la que los 24 participantes completaron las dos planillas que se les ofrecieron. Las razones de esta decisión se explican en el apartado Discusión.

Para el Fragmento 1,12 participantes eligieron la palabra crescendo, 9 participantes emergiendo, respirado y rítmico, 8 participantes abrupto, enérgico y expandido, 6 participantes fluido y 5 participantes fuerte, impulso, saltando, suave y vigoroso. La Figura 2 muestra mediante una nube todas las palabras que fueron seleccionadas para describir el Fragmento 1, sumando ambas condiciones (sábado y domingo).

El Fragmento 2 fue correlacionado por 12 participantes con la palabra respirado, por 9 con suspendido y por 7 con tenso. La Figura 3 muestra mediante una nube todas las palabras que fueron seleccionadas para describir el Fragmento 2, sumando ambas condiciones (sábado y domingo). 


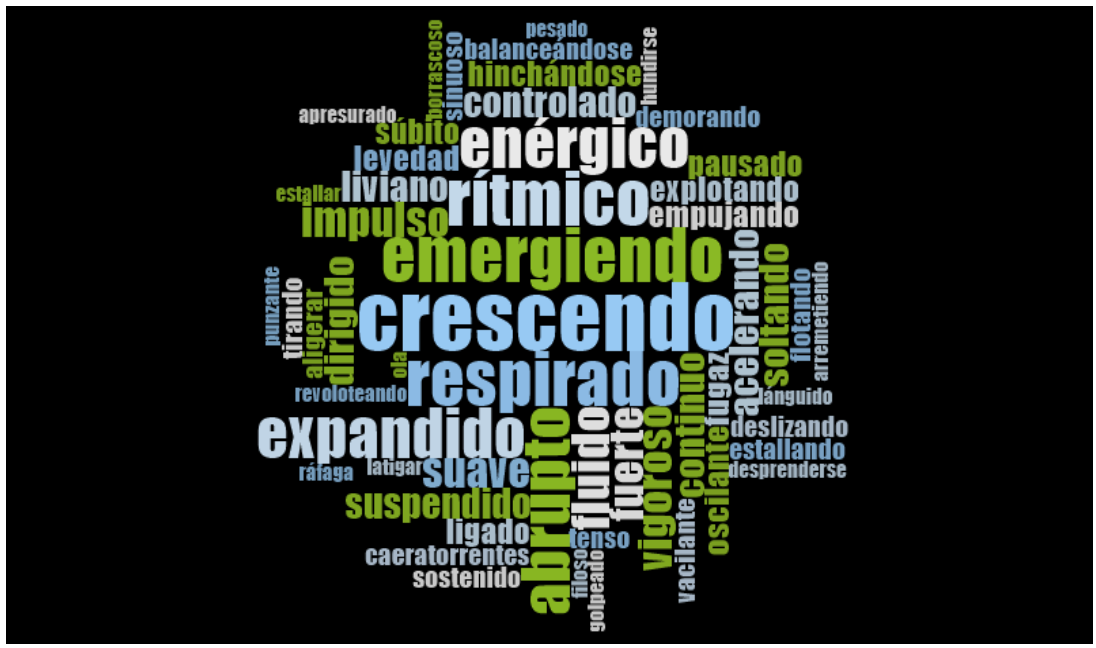

Figura 2. Total de palabras seleccionadas para describir el Fragmento 1 sumando ambas condiciones. El tamaño de las palabras en el gráfico se corresponde con la frecuencia en que fueron elegidas.

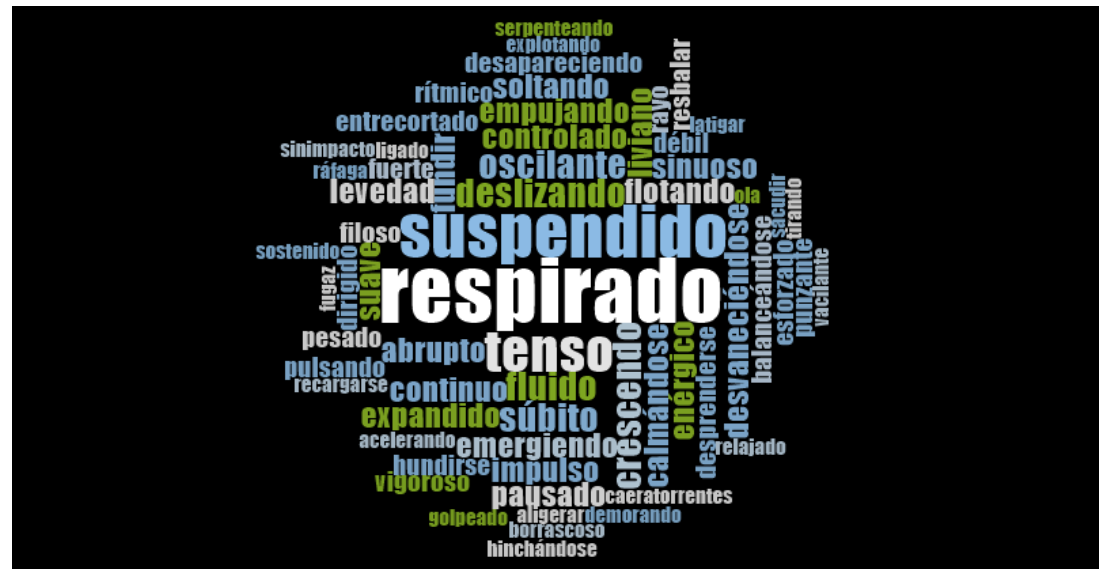

Figura 3. Total de palabras seleccionadas para describir el Fragmento 2 sumando ambas condiciones. El tamaño de las palabras en el gráfico se corresponde con la frecuencia en que fueron elegidas.

Si ahora comparamos cómo se percibieron los Fragmentos 1 y 2 tomando solamente las palabras más elegidas y siempre sumando ambas condiciones (ver Fig. 4), la categoría crescendo, atribuida al Fragmento 1 por 12 participantes, fue elegida para el Fragmento 2 sólo por 4 participantes. La categoría emergiendo, elegida 
para el Fragmento 1 por 9 participantes, fue elegida para el Fragmento 2 sólo por 4. El Fragmento 2, por su parte, fue descrito como suspendido por 9 participantes, mientras que el Fragmento 1 tuvo esa cualidad sólo para 4 personas. La cualidad de respirado fue la más pareja: fue asignada al Fragmento 2 por 12 participantes y al Fragmento 1 por 9; es decir, ambos fragmentos se consideraron respirados.

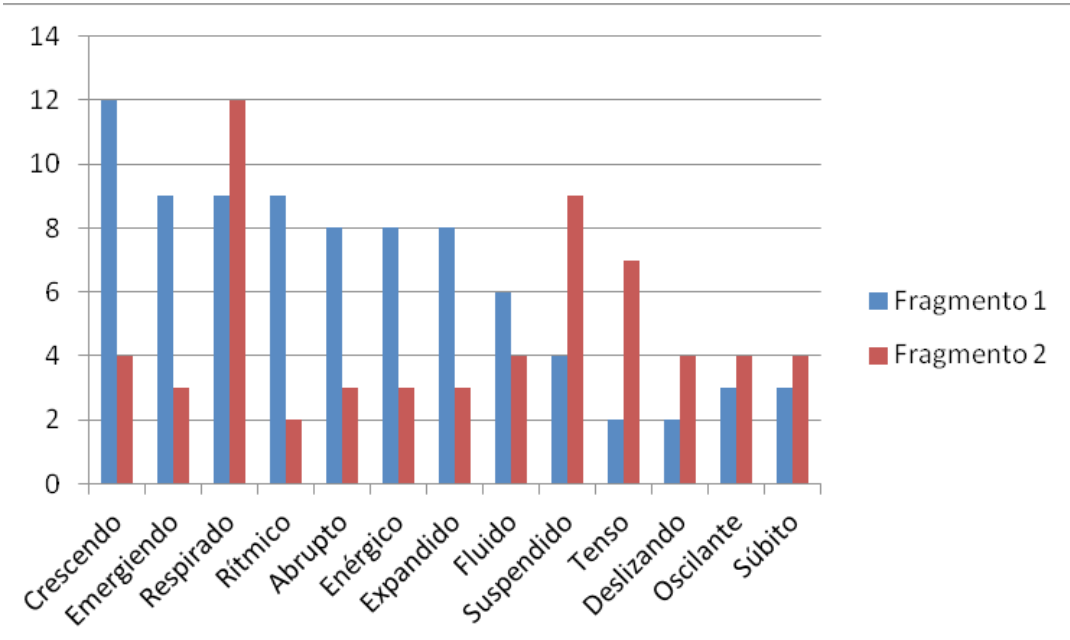

Figura 4. Se comparan los dos fragmentos tomando como referencia únicamente las palabras más elegidas en ambas condiciones juntas.

Si ahora miramos los resultados discriminándolos por condición y por fragmento (ver Tabla 1) observamos que: en la Condición A, para el Fragmento 1, 9 participantes eligieron crescendo, 7 enérgico, 6 respirado, 5 abrupto y emergiendo, 4 expandido, fuerte, rítmico y vigoroso. (También eligieron otras palabras, pero en estas coincidió ese número de participantes). Mientras que para el Fragmento 2, en la misma condición, 8 eligieron respirado, 7 suspendido, 5 tenso y 3 crescendo, deslizando y suave".

En cambio, en la Condición B para el Fragmento 1 el mayor número de coincidencias fue rítmico y fue sólo de 5 participantes, seguido de emergiendo y expandido elegida por 4 participantes. Para el Fragmento 2, el mayor número de coincidencias fue para respirado, palabra que, de todos modos, reunió sólo 4 participantes.

$\mathrm{Si}$ ahora comparamos ambas condiciones, vemos que los participantes de la Condición A (sábado) tuvieron mucha mayor coincidencia entre sí en las palabras elegidas para ambos fragmentos que los participantes de la Condición B (domingo). Más allá de la evidente dispersión de datos en la Condición B en comparación 
Epistemus - volumen 8 - número 1 (Julio de 2020)

DOI: https://doi.org/10.24215/18530494e 013

con la Condición A, las diferencias en cómo se percibió cada fragmento en una y otra condición saltan a la vista en las Figuras 5 y 6.

\begin{tabular}{|c|c|c|}
\hline & Fragmento 1 & Fragmento 2 \\
\hline \multirow[t]{17}{*}{ Condición A } & Crescendo (9) & Respirado (8) \\
\hline & Enérgico (7) & Suspendido (7) \\
\hline & Respirado (6) & Tenso (5) \\
\hline & Abrupto (5) & Crescendo (3) \\
\hline & Emergiendo (5) & Deslizando (3) \\
\hline & Expandido (4) & Suave (3) \\
\hline & Fuerte (4) & \\
\hline & Rítmico (4) & \\
\hline & Vigoroso (4) & \\
\hline & Controlado (3) & \\
\hline & Dirigido (3) & \\
\hline & Empujando (3) & \\
\hline & Fluido (3) & \\
\hline & Impulso (3) & \\
\hline & Liviano (3) & \\
\hline & Pausado (3) & \\
\hline & Suspendido (3) & \\
\hline \multirow[t]{9}{*}{ Condición B } & Rítmico (5) & Respirado (4) \\
\hline & Emergiendo (4) & Fluido (3) \\
\hline & Expandido (4) & \\
\hline & Abrupto (3) & \\
\hline & Crescendo (3) & \\
\hline & Fluido (3) & \\
\hline & Respirado (3) & \\
\hline & Saltando (3) & \\
\hline & Suave (3) & \\
\hline
\end{tabular}

Tabla 1. Cuadro comparativo de las palabras más seleccionadas para cada fragmento en cada condición. Los números entre paréntesis indican la frecuencia de elección. Se omiten en este cuadro las palabras no seleccionadas y las palabras seleccionadas 1 y 2 veces.

El Fragmento 1 fue percibido como crescendo por 9 participantes de la Condición A (versus 3 de la Condición B). Fue percibido como enérgico por 7 participantes de la Condición A (y por sólo 1 de la Condición B). Fue percibido como respirado por 6 participantes de la Condición A (versus 3 de la Condición B). Y lo percibieron emergiendo, abrupto, rítmico y expandido en números similares entre ambas condiciones (ver Figura 5). 


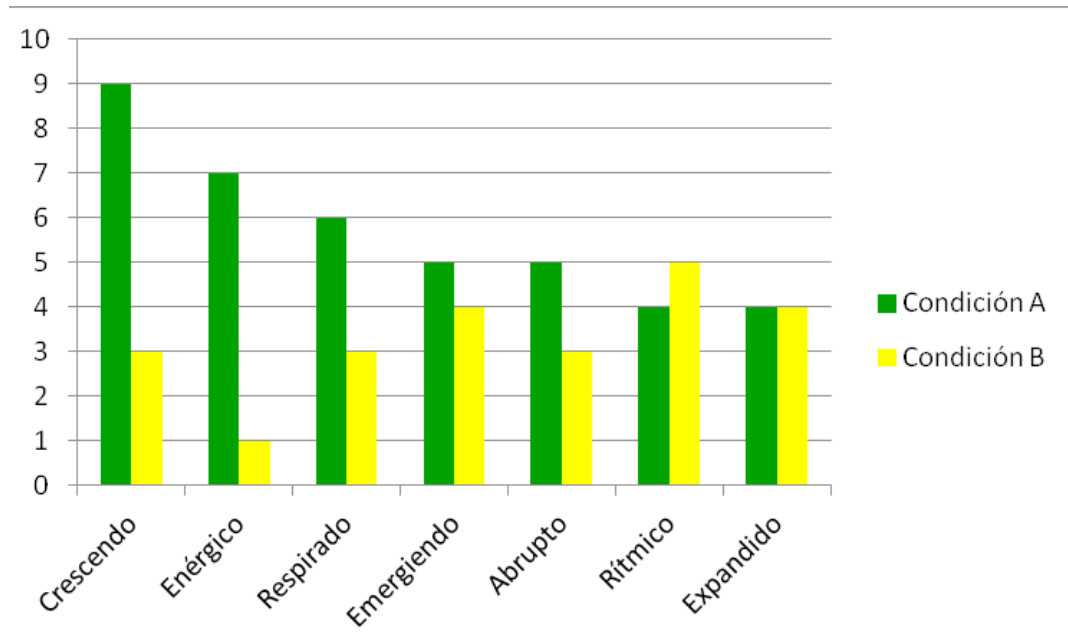

Figura 5. Palabras más elegidas para describir el Fragmento 1, comparando ambas condiciones.

El Fragmento 2 fue percibido como respirado por 8 participantes de la Condición A (versus cuatro de la Condición B), como suspendido por 7 participantes de la A (versus 2 de la Condición B), como tenso por 5 participantes de la Condición A (versus 2 de la Condición B) y como crescendo y deslizando por 3 participantes de la Condición A (versus 1 de la Condición B). Fue también percibido como fluido por 3 participantes de la Condición B (versus sólo 1 de la condición A) (ver Figura 6).

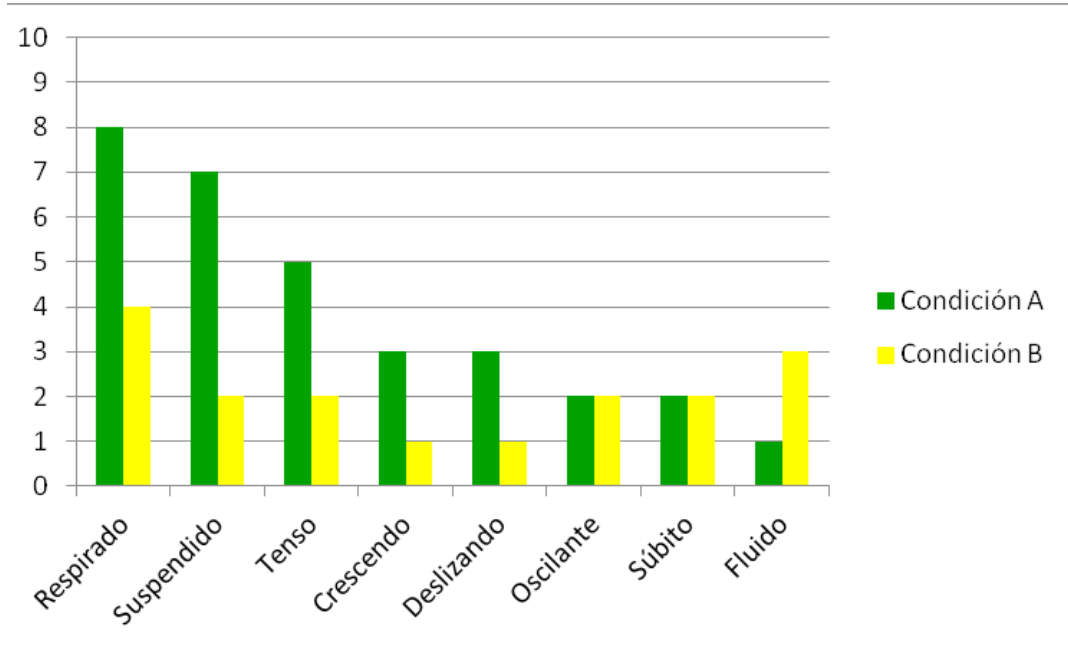

Figura 6. Palabras más elegidas para describir el Fragmento 2, comparando ambas condiciones. 
No es posible saber qué significa la mayor dispersión de datos en la Condición B. De todos modos, pareciera que la percepción de las formas de la vitalidad en los fragmentos se vio influida por las experiencias inmediatamente anteriores. Es decir, el hecho de que para 9 participantes de la Condición A el Fragmento 1 fuera crescendo y para 7 enérgico, mientras que los participantes de la Condición B lo vieran de diversas maneras -y crescendo fuera elegida sólo por 3- nos habla de que muy posiblemente las propias condiciones hayan influido en esa captación.

Las diferencias en la percepción de un mismo fragmento podrían atribuirse al tipo de movimientos corporales realizados en la Estación a (movimiento) con anterioridad a esta tarea, y al estímulo musical recibido de manera repetitiva en cada condición. En este sentido, resulta necesario caracterizar brevemente el tipo de movimientos realizados por los participantes en la tarea de movimiento, que quedaron registrados en video tal como se describe en el apartado Procedimiento.

Los movimientos que realizaron los participantes el día sábado (Condición A) son mayormente amplios (especialmente movimientos abarcativos de brazos), expandidos, enérgicos, con giros, impulsos y desplazamientos.

El día domingo (Condición B) los participantes desplegaron en general mucha menor cantidad de movimientos, y éstos se caracterizaron por ser más lentos, con varios momentos de quietud o casi quietud, uso casi exclusivo de la posición vertical con algunos movimientos hacia abajo y arriba sobre el eje vertical, pocos desplazamientos, movimientos de brazos y torso más bien cerrados (hacia el propio cuerpo), y notable frecuencia de gestos de manos hacia cara o cabeza.

Resulta una explicación plausible que el realizar estos movimientos, y el verse expuestos a un estímulo musical determinado (contrastante entre Condiciones A y B), haya influido en la captación de formas de la vitalidad del fragmento, generando diferencias entre ambas condiciones. Aquí la hipótesis sería una de cognición corporizada: la percepción de las formas de la vitalidad se vio influida por el estado corporal predominante al realizar la tarea.

\section{Discusión}

Esta investigación, parte de cuyos resultados se presentan y discuten aquí, se topó con ciertas dificultades propias del objeto de estudio: por un lado, el desafío de estudiar eventos dinámicos cuya captación es mayormente implícita y se produce sin la intervención del lenguaje; y por el otro, la dificultad propia de un estudio en el marco de un evento teatral, es decir de un acontecimiento efímero que involucra elementos no controlables por la investigación.

Antes de adentrarnos en la discusión de los resultados que presentamos, resulta necesario comentar un aspecto de lo que sucedió en la función del día do- 
mingo, un hecho no previsto que pudo haber modificado en parte la experiencia. Esa noche, mientras sucedía la función, Boca jugó contra River en el clásico de la Superliga, un partido muy importante para los numerosos amantes del fútbol en todo el país. La sala donde se realizaba la función está ubicada en la plaza central de la ciudad, donde habitualmente se festejan goles y suceden diferentes actos políticos y culturales. Mientras transcurría la función, y especialmente en momentos de silencio de la obra, se escuchaban los gritos festejando los goles, lo que inevitablemente pasó a ser parte del acontecimiento, y, según pudimos corroborar posteriormente, fue vivido de maneras muy diferentes por espectadores de distintas generaciones. Para las personas más jóvenes, esto fue un ruido que los perturbaba, y los perturbaba bastante. Para las personas de más edad, incluidos algunos de los investigadores, que ya éramos adultos o jóvenes en la época de la dictadura, esta intromisión sonora retrotrajo la experiencia al momento del Mundial de Fútbol 78 en Argentina, en el que el país festejaba los goles mientras la dictadura cívicomilitar cometía los más horrendos crímenes. La resonancia de esta simultaneidad con el tema de la obra Dibaxu cargó de nuevos sentidos la experiencia para los espectadores de mayor edad. Cabe la posibilidad de que la interferencia provocada por esos sonidos tan fuertes haya influido en el hecho de que las personas percibieran los fragmentos de manera bastante más disímil entre ellas que el día anterior. Por otro lado, ese domingo el grupo de teatro, proveniente de la ciudad de Viedma, realizaba la última función de esta obra luego de muchos años. Al final de la función, el director subió al escenario y emocionado comentó esta situación. Es decir, entonces, que el día domingo hubo dos factores que se agregaron a la experiencia de presenciar esta obra, que no estuvieron presentes el día sábado.

El realizar estudios de este tipo en sus contextos naturales o ecológicos (en este caso, la función teatral a la que asisten otros espectadores no incluidos en el estudio) presenta ventajas y desventajas. Por un lado los resultados no devienen de situaciones experimentales artificiales, cuya traspolación a la vida cotidiana puede ser problemática, sino que se recuperan aspectos de las situaciones reales. La contrapartida es la imposibilidad de controlar todas las variables, del modo en que sí puede hacerse en situaciones artificiales. Esto sucedió en este estudio, donde no fue posible controlar todas las variables precisamente por tratarse de teatro, un hecho vivo donde en cada función pueden suceder y de hecho a menudo suceden imprevistos.

Quisiéramos por otro lado incluir aquí una reflexión evaluando a posteriori aspectos del método. Se volvió claro que el método diseñado e implementado, al involucrar varias tareas, dos condiciones y dos fragmentos como estímulos, resultó tal vez excesivamente complejo, sumado esto a la diversidad de factores que implica estudiar la experiencia en el marco de una función teatral. Esta misma complejidad y la novedad del diseño hicieron asimismo difícil prever con exhaus- 
tividad cómo se analizarían la totalidad de los datos recogidos, lo cual deberá corregirse en futuros estudios de este tipo.

Otro punto que merece ser nombrado aquí se refiere a la utilización de palabras como descriptores de formas de la vitalidad. Si bien es cierto que las formas de la vitalidad son captadas y a menudo compartidas de modo implícito no verbal, consideramos importante poder indagar en el aspecto lingǘstico de esta experiencia. El ya citado estudio de Martínez y Pereira Ghiena (2011) también dio por supuesto que las formas de la vitalidad emergentes de la música y el movimiento podían asociarse a las descripciones lingüísticas que dan cuenta de esa calidad vital. En línea con dicha investigación, se consideró la palabra descriptora de una forma de la vitalidad como comunicación de la experiencia sentida.

$\mathrm{Al}$ igual que en otros estudios, se eligieron ciertas palabras para nombrar las formas de la vitalidad; no se ofreció a los participantes una explicación sobre el significado de las palabras, confiando en la comunalidad de significados compartidos entre parlantes del español argentino. El objetivo no fue tanto determinar la correspondencia específica y puntual entre cada forma de la vitalidad y una palabra, sino el grado en que los participantes coincidían en elegir las mismas o similares palabras para nombrarlas. No hay certeza acerca de las correspondencias entre formas de la vitalidad y palabras descriptoras, pero frente a la opción de simplificar excesivamente la tarea tomando sólo pares de palabras básicas (tipo fuertedébil) se prefirió ofrecer y obtener mayor riqueza de descripciones, a riesgo quizás de una mayor imprecisión. A su vez esto acarreó un problema metodológico: la dispersión de los datos. Será necesario corregir este aspecto al repetir el estudio, siendo una solución posible el limitar el número de opciones que los participantes pueden seleccionar.

Así como Martínez y Pereira Ghiena (2011) lo hicieron con respecto a la música, este estudio buscó estudiar la captación de las formas de la vitalidad en el teatro a partir de descripciones lingüísticas de dicha experiencia. Sin embargo, una diferencia del presente trabajo respecto del citado es que previamente a la tarea que involucra una descripción lingüística (Estación de palabras), todos los participantes realizaron dos tareas que involucraban descripciones no-lingüísticas, como el movimiento corporal explícito (Estación de movimiento) y la expresión gráfica (Estación de dibujos). En tal sentido, estas descripciones no lingüísticas implicaron momentos de pasar por el cuerpo directamente; posteriormente realizaban la tarea de conceptualización, habiendo transitado esas tareas de experiencia sentida.

Las formas de la vitalidad son captadas de manera implícita, no verbal, y al estudiarlas con tareas que involucran el nombrarlas se genera una cierta contradicción. Al diseñar el estudio, elegimos gran número de palabras descriptoras de formas de la vitalidad para poder así reflejar mayores sutilezas de una experien- 
cia particularmente evasiva para ser captada a través del lenguaje. A la hora de analizar los resultados, nos encontramos con que el gran número de palabras, y el que la consigna no estableciera un máximo a elegir, produjo dispersión en los datos, como ya se mencionó. Sin embargo, y a pesar de que las palabras entre las cuales podían elegir los sujetos eran muchas (75), los resultados muestran una confluencia en palabras para describir los fragmentos, y esta coincidencia estaría confirmando la captación de las formas de la vitalidad, así como ciertos consensos en cómo nombrarlas o describirlas con palabras. Los sujetos eligen para describir el Fragmento 1 mayormente las palabras crescendo(12), emergiendo, enérgico, respirado y rítmico (8), y para describir el Fragmento 2, respirado (10), suspendido(9) y tenso (7).

En el intento de superar las limitaciones para nombrar las formas de la vitalidad, en este estudio se buscó complementar las consignas que implicaban el uso del lenguaje con otras no lingüísticas (movimiento y dibujo). La razón por la que en el presente artículo se presentan únicamente los resultados de la tarea de palabras es que cada una de las tareas realizadas arrojó resultados que requieren un método de análisis propio. Por ejemplo, las filmaciones de las pequeñas escenas producidas por los participantes en la tarea de movimiento deberán ser analizadas con un programa de análisis de movimiento y requerirán la creación de categorías que permitan un análisis transversal. El plan del equipo de investigación es presentar trabajos separados donde se analizan los resultados de cada una de las pruebas, y luego un artículo final donde se relacionarán todos, para aportar a este reciente campo de estudios mayores conocimientos y solidez.

En cuanto al estudio de la obra viva, éste se topa con diversos grados de imprevisibilidad que conllevan los fenómenos que suceden: las cosas cambian, no siempre suceden igual. Una función de teatro danza es un evento múltiple y socio-cultural, constituido por diversos aspectos, como los bailarines, la música, el vestuario, la iluminación, la escenografía, los demás espectadores, etcétera, que conjuntamente impactan al espectador (Jola, Ehrenberg y Reynolds, 2012).

El dilema metodológico y teórico de este tipo de estudios reside en que cuanto más simple es el diseño y menor la cantidad de variables involucradas, más sencillo resulta obtener resultados claros, pero a la vez se corre el riesgo de simplificar en extremo la riqueza de un fenómeno complejo y multidimensional, como es una obra teatral. En el trabajo aquí relatado se decidió utilizar un estímulo o serie de estímulos de por sí complejos (el espectáculo y los fragmentos del espectáculo) y se estudió la percepción a través de tareas también complejas. A la hora de analizar los resultados, vemos que debido a la secuenciación de tareas en la prueba, la elección de las palabras para caracterizar los fragmentos puede haber sido no solamente resultado de la experiencia directa de la percepción de los fragmentos, sino resultado de esa percepción sumada a la experiencia del propio movimiento corporal posterior en simultáneo con un estímulo sonoro constante. A la par, la 
recepción de la obra viva puede implicar la intervención de fenómenos sociales no previstos, como sucedió en este caso con los gritos festejando goles que resonaron de manera diferenciada en los participantes en una de las condiciones.

Por último, y más allá de los objetivos del estudio, la imprevisibilidad de la obra viva regaló un fenómeno que vale la pena destacar: los espectadores que fueron sujetos de la investigación sintieron, según relataron en el círculo final, que las tareas realizadas modificaron y completaron la experiencia de presenciar la obra Dibaxu. La elaboración de lo vivido que habitualmente acompaña una experiencia de este tipo y que suele realizarse en conversación con otros o en soledad, se produjo en este caso en un despliegue de re-creaciones posteriores compartidas en múltiples niveles: de lenguaje, de movimiento, de expresión visual y de conversación grupal. El corte brusco que se produce al finalizar una obra y retornar a la vida cotidiana en esta ocasión se vio tamizado por una experiencia de elaboración conjunta que, en palabras de los participantes, modificó beneficiosamente y completó la experiencia de presenciar la obra teatral. Sin proponérselo, el diseño de investigación se transformó en un dispositivo de acompañamiento y sostén compartido de la elaboración de la recepción teatral.

\section{Notas}

1. Alicia Clara Nudler: http://orcid.org/0000-0002-2142-8693

2. María de la Paz Jacquier: http://orcid.org/0000-0002-9183-5307

3. Silvia Español: http://orcid.org/0000-0003-3039-0698

4. Este artículo es resultado del proyecto de investigación Aportes al análisis teatral desde la psicología cognitiva corporeizada de la Universidad Nacional de Río Negro.

\section{Agradecimientos}

Agradecemos al director teatral Hugo Aristimuño y los actores de Teatro del Viento Débora Sansó, Carlos Irazusta y Guillermo Riegelhaupt por colaborar de manera entusiasta con la implementación de la experiencia de investigación.

Agradecemos a los integrantes del equipo de investigación Adrián Porcel de Peralta, Sol Alonso, Martín Contuci y Agustín Schmeisser por su colaboración en la producción de la obra Dibaxu y el diseño e implementación del estudio. 


\section{Referencias}

Amiina. (2007). Boga. En Kurr [Álbum]. Mosfellsbaer: Bláskjár Records.

Aristimuño, H. (2015). En M. Tossi (Comp.) Antología de teatro rionegrino en la postdictadura. 127-140. Viedma: Universidad Nacional de Río Negro.

Beckett, S. (1986) Krapp’s Last Tape. En Samuel Beckett The complete dramatic works. 213-223. Londres: Faber and Faber. (Primera edición de esta obra: 1958).

Bussières, N. (2018). Light, vitality, and dynamism. An introduction of time and movement into theatre lighting design. En Thesis Presented for the Partial Fulfillment of the Requirements For the Degree of Master of Arts (Individualized Program). Concordia University, Montreal.Di Cesare, G.; Di Dio, C.;Rochat, M.; Sinigaglia, C.;Bruschweiler-Stern, N.; Stern, D. y Rizzolatti, G. (2013). The neural correlates of vitality form recognition: an fMRI study. Social Cognitive and Affective Neuroscience, 9(7), 951-960. DOI 10.1093/scan/ nst068

Español, S. y Bordoni, M. (2018). Antecedentes del juego con las formas de la vitalidad al inicio del segundo año de vida. En N. Alessandroni y M. I. Burcet (Comp.), La experiencia musical: investigación, interpretación y prácticas educativas. Actas del $13^{\circ}$ Encuentro de Ciencias Cognitivas de la Música, 76-89. Buenos Aires: SACCOM.

Español, S., Bordoni, M., Martínez, M. y Carretero, S. (2015). Forms of vitality play and symbolic play during the third year of life. Infant Behavior and Development, 40, 242-252. DOI 10.1007/s12124-014-9271-5

EuroArtsChannel (2002). Bobby McFerrin: Vivaldi - Concerto for two cellos in g minor, RV 531 (Gewandhausorchester Leipzig). [Video] Youtube. https://youtube.com/ watch? $\mathrm{v}=\mathrm{ip} 09 \mathrm{OPFfJb} 8$

Galeano, E. (2013) El libro de los abrazos. Buenos Aires: Siglo XXI. (Primera edición de esta obra: 1989)

Jola, C., Ehrenberg, S. y Reynolds, D. (2012). The experience of watching dance: Phenomenological-neuroscience duets. Phenomenology and the Cognitive Science. 11, 17-37. DOI 10.1007/x11097-010-9191-x

Jones, A. (2012). Foreword: Kinesthetic empathy in philosophical and art history: Thoughts on how and what art means. En Reynolds, D. and Reason, M. (Eds.), Kinesthetic Empathy in Creative and Cultural Practices. Bristol UK: Intellect.

Leman, M. (2008). Embodied Music Cognition and Mediation Technology. Cambridge: the MIT Press. [Cognición musical corporeizada y tecnología de mediación. (I. C. Martínez, F. Shifres, C. Mauleón, V. Silva, D. Callejas Leiva y R. Herrera, trads.) Buenos Aires: SACCoM, 2011.] 
Martínez, I. y Pereira Ghiena, A. (2011). La experiencia de la música como forma vital. Perfil dinámico temporal, corporalidad y forma sónica en movimiento. En Pereira Ghiena, A. Jacquier, P., Valles, M. y Martínez, M. (Eds.), Musicalidad Humana: Debates actuales en evolución, desarrollo y cognición e implicancias socio-culturales. Actas del X Encuentro de Ciencias Cognitivas de la Música, 521-530. Buenos Aires: SACCOM.

Nudler, A., Español, S., Jacquier, M. de la P. y Porcel de Peralta, J. A. (2018). Un análisis de las formas de la vitalidad en la obra La última cinta de Krapp. Epistemus, 6, 2, 122 130. DOI 10.21932/epistemus.6.6186.2

Rochat, M., Veroni, V., Bruschweiler-Stern, N., Pieraccini, C. Bonnet-Brilhault, F., Barthélémy, C., Malvy, J., Sinigaglia, C., Stern, D. y Rizzolatti, G. (2013). Impaired vitality form recognition in autism. Neuropsychologia, 51, 1918-1924. DOI 10.1016/j. neuropsychologia.2013.06.002

Shifres, F. (2008). Música, transmodalidad e intersubjetividad. Estudios de Psicología, 29(1), 7-30. DOI: $10.1174 / 021093908783781347$

Shifres, F., Pereira Ghiena, A., Herrera, R. y Bordoni, M. (2012). Estilo de ejecución musical y de danza en el tango: atributos, competencia y experiencia dinámica. Cuadernos de Música, Artes Visuales y Artes Escénicas, 7, (2/Julio - Diciembre de 2012), 83-108. Bogotá, D.C., Colombia.

Stern, D. ([1985] 2005). El mundo interpersonal del infante. Una perspectiva desde el psicoanálisis y la psicología evolutiva. Buenos Aires: Paidós (Primera edición en inglés: 1985).

Stern, D. (2004). The Present Moment in Psychotherapy and Everyday Life. New York: Norton.

Stern, D. (2010). Forms of Vitality: Exploring Dynamic Experience in Psychology, the Arts, Psychotherapy, and Development. Oxford: University Press.

The Watching Dance Project (2008-2011). Watching Dance: Kinesthetic Empathy. Recuperado el 20 de setiembre de 2019 de http:/ / www.watchingdance.org/index.php

Trondalen, G. y Skårderud, F. (2007) Playing with affects... and the importance of affect attunement. Nordic Journal of Music Therapy, 16(2) 2007, 100-111. 